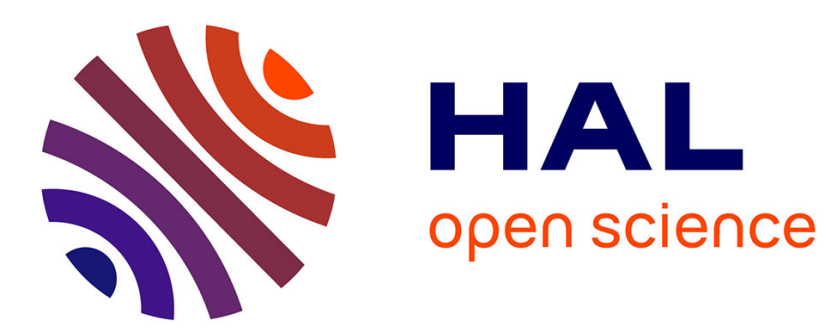

\title{
Collision avoidance in low thrust rendezvous guidance using flatness and positive B-splines
}

Christophe Louembet, Georgia Deaconu

\section{To cite this version:}

Christophe Louembet, Georgia Deaconu. Collision avoidance in low thrust rendezvous guidance using flatness and positive B-splines. American Control Conference 2011 (ACC 2011), Jun 2011, San Francisco, United States. 6p. hal-00526269

\section{HAL Id: hal-00526269 \\ https://hal.science/hal-00526269}

Submitted on 14 Oct 2010

HAL is a multi-disciplinary open access archive for the deposit and dissemination of scientific research documents, whether they are published or not. The documents may come from teaching and research institutions in France or abroad, or from public or private research centers.
L'archive ouverte pluridisciplinaire HAL, est destinée au dépôt et à la diffusion de documents scientifiques de niveau recherche, publiés ou non, émanant des établissements d'enseignement et de recherche français ou étrangers, des laboratoires publics ou privés. 


\title{
Collision avoidance in low thrust rendezvous guidance using flatness and positive B-splines
}

\author{
Christophe Louembet* \\ ${ }^{*}$ CNRS ; LAAS ; 7 avenue du colonel Roche, F-31077 Toulouse, \\ France \\ Université de Toulouse ; UPS, INSA, INP, ISAE ; LAAS ; F-31077 \\ Toulouse, France
}

\begin{abstract}
Collision avoidance issue in close proximity orbital rendezvous is addressed in this paper. The arising optimal control problem is solved by means of differential flatness in order to apply algebraic geometry tools. These tools based on B-splines parametrization and positive piecewise polynomial concept provide a certification of constraints satifaction continuously in time contrary to classical collocation techniques. The non convexity derived from the avoidance constraint is overcome by using time-varying convex approximation.
\end{abstract}

Keywords: Collision avoidance, Orbital rendezvous, Continuous thrust, Trajectory planning, Differential Flatness, B-spline positivity

\section{INTRODUCTION}

Spacecraft rendezvous is an enabling technology for present and future space missions.

In recent years, electric propulsion has been used and proposed for many space applications including orbit transfers and station keeping (see Anzel [1988, 1998], Gopinath and Srinivasamuthy [2003]), but also for interplanetary and deep space missions (see Rayman et al. [1999], GilFernndez et al. [2006]). In this paper, we exploit direct methods that can easily handle linear and non linear path constraints on contrary to the Pontryagin's maximum principle methods,. These methods, based on parametrization and/or discretization of the original optimal control problem (see Hull [1997]) have been successful in solving both impulsive and continuous thrust rendezvous problem under path constraints (see for instance Breger and How [2008] for impulsive maneuver and Armellin et al. [2004] for electric propulsion and the references therein).

In this paper, the focus is on the collision avoidance issue in close proximity maneuver. This issues has been studied from different point of view for both impulsive and continuous thrust. A popular approach in path planning community is the artificial potential techniques that consists in completing the dynamics of the flying formation with a force derived from a potential such the two spacecraft are repelled from each others, Junge and Ober-Blobaum [2005]. Another strategy to ensure a safety distances between spacecrafts is to add to the cost function a penaty term, Junge and Ober-Blobaum [2005], Armellin et al. [2004]. These two approaches can be related to the electrostatic collision avoidance technique developed by Wang and Schaub [2010] where the spacecraft are charged with positive or negative electric potential and take advantage of the derived Coulomb force.

On the other side, collision avoidance can be addressed by adding avoidance constraints to parametric optimisation problem and applying the pertinent programming solver. Thus, early works from Eckstein et al. [1989] consisted in solving the avoidance constraints with non linear programming solver. Unfortunately, from the non convexity of the avoidance problem follows the existence of multiple local minima. Richards et al. [2002] addressed this drawback in the framework of linear Hill-Chlohessy-Wiltshire relative motion model by enforcing of a rectangular exclusion region around vehicles and using the mixed integer linear programming. Finally, in order to obtain a convex program, the exclusion region constraints has been replaced by a rotating convex approximation in Mueller and Larsson [2008], Breger and How [2008]. The late papers are interesting since they imply convex linear programs that are known to be computationnally tractable. We propose, in this paper, a methodology based on differential flatness (see Fliess et al. [1992]) that is able to ensure constraints satisfaction all along the path on the contrary to classical direct methods as developped in Mueller and Larsson [2008], Breger and How [2008].

Using differential flatness, eligible optimal control problems could be solved by means of geometric techniques (and thus avoiding integration of the dynamics) using the flat trajectory parametrization. In the differential flatness context, a classical and tractable methodology developped in Milam et al. [2000], Faiz et al. [2001], Louembet et al. [2009] relies on B-splines based collocation. However, as in classical direct methods, this technique involves time sampling: No guarantee on constraints satisfaction between collocation points can be ensured. This may lead to critical issues that need to be detected by an appropriate post-analysis (see examples from Breger and How [2008], Louembet et al. [2009] for instance). In this paper, our goal is to design flat system trajectories, using the convenient B-splines parametrization, that guarantee continuous con- 
straints satisfaction in time and without the need for a post-analysis.

Using the results from Louembet et al. [2010] on positive piecewise polynomials, the main result of this paper is to provide a new motion planning methodology that allows to design a trajectory fulfilling the constraints continuously in time.

In section 2 , we briefly present the relative motion model and the concept of differential flatness. Then, the optimal path planning problem for flat systems is described. Our contribution is detailed in section 3 in two steps. First, in subsection 3.1, the results on positive polynomials from Louembet et al. [2010] is presented. Subsequently, in subsection 3.2, the constrained B-splines optimization is formulated as a convex optimization problem over linear matrix inequalities (LMI) for which efficient programming (SDP) solvers are available. In section 4 , an example of the resolution of an orbital homing problem illustrates the methodology.

\section{PROBLEM STATEMENT}

\subsection{Relative motion model}

In this paper, the Rendezvous mission consists of two spacecraft: one chaser satellite with full 3-axis capability and one passive target spacecraft on an arbitrary elliptic orbit. This particular relative motion between the two satellites in close space was firstly described in the space context in Tschauner [1966]. In the following, we describe briefly the developement of the equations of motions in order to obtain a state model of the relative dynamics.

In the context of an elliptic keplerian orbit, the linearized relative motion is stated by the so-called Tshauner-Hempel equations:

$$
\left\{\begin{aligned}
\ddot{x}= & 2 n \frac{(1+e \cos \nu)^{2}}{\left(1-e^{2}\right)^{3 / 2}} \dot{y}-2 n^{2} e \sin \nu\left(\frac{1+e \cos \nu}{1-e^{2}}\right)^{3} y \ldots \\
& +n^{2}\left(\frac{1+e \cos \nu}{1-e^{2}}\right)^{3}(3+e \cos \nu) x+n^{2} u_{R} \\
\ddot{y}= & -2 n \frac{(1+e \cos \nu)^{2}}{\left(1-e^{2}\right)^{3 / 2}} \dot{x}+2 n^{2} e \sin \nu\left(\frac{1+e \cos \nu}{1-e^{2}}\right)^{3} x \\
& +n^{2}\left(\frac{1+e \cos \nu}{1-e^{2}}\right)^{3}(e \cos \nu) y+n^{2} u_{S} \\
\ddot{z}= & -n^{2}\left(\frac{1+e \cos \nu}{1-e^{2}}\right)^{3} z+n^{2} u_{W}
\end{aligned}\right.
$$

where $\nu$ is the true anomaly, $e$ the excentricity, $n$ the mean motion and the input vector $u(t)$ is derived from the propulsion force $F_{\text {chaser }}$ and the $m_{\text {chaser }}$ :

$$
\left\{\begin{array}{l}
u_{R}=\frac{F_{\text {chaser }, R}}{m_{\text {chaser }} n^{2}} \\
u_{S}=\frac{F_{\text {chaser }, S}}{m_{\text {chaser }} n^{2}} \\
u_{W}=\frac{F_{\text {chaser }, W}}{m_{\text {chaser }} n^{2}}
\end{array}\right.
$$

Simplified Tschauner-Hempel equations can be obtained by replacing time as the independent variable by the true anomaly, $\nu$, and by using a classical change of variables (see Tschauner [1966] for detail):

$$
\left\{\begin{array}{l}
\tilde{x}^{\prime \prime}=2 \tilde{y}^{\prime}+\frac{3}{1+e \cos \nu} \tilde{x}+\tilde{u}_{R} \\
\tilde{y}^{\prime \prime}=-2 \tilde{x}^{\prime}+\tilde{u}_{S} \\
\tilde{z}^{\prime \prime}=-\tilde{z}+\tilde{u}_{W}
\end{array}\right.
$$

We can now define the state vector $\tilde{X}(\nu)=\left[\begin{array}{llllll}\tilde{x} & \tilde{y} & \tilde{z} & \tilde{x}^{\prime} & \tilde{y}^{\prime} & \tilde{z}^{\prime}\end{array}\right]$ and the associated input vector $\tilde{u}=\left[\begin{array}{lll}\tilde{u}_{R} & \tilde{u}_{S} & \tilde{u}_{W}\end{array}\right]$ and deduce the linear time-periodic state space model:

$$
\frac{d \tilde{X}(\nu)}{d \nu}=\tilde{A}_{T H} \tilde{X}(\nu)+\tilde{B}_{T H} \tilde{u}(\nu)
$$

with:

$$
\tilde{A}_{T H}=\left[\begin{array}{cccccc}
0 & 0 & 0 & 1 & 0 & 0 \\
0 & 0 & 0 & 0 & 1 & 0 \\
0 & 0 & 0 & 0 & 0 & 1 \\
3 & 0 & 0 & 0 & 2 & 0 \\
\frac{3+e \cos \nu}{1+{ }_{0}} & 0 & 0 & -2 & 0 & 0 \\
0 & 0 & -1 & 0 & 0 & 0
\end{array}\right] \tilde{B}_{T H}=\left[\begin{array}{lll}
0 & 0 & 0 \\
0 & 0 & 0 \\
0 & 0 & 0 \\
1 & 0 & 0 \\
0 & 1 & 0 \\
0 & 0 & 1
\end{array}\right]
$$

\subsection{Differential flatness}

Differential flatness, or flatness in short, has been introduced Fliess et al. [1992] in 1992. Consider a nonlinear system:

$$
\dot{X}=f(X, u)
$$

where $X$ is the $n$-component state vector and $u$ the $m$ component control assuming that $m \leq n$.

Definition 1. The nonlinear system (6) is differentially flat if there exists an $m$-dimensional vector $\chi$, whose elements are differentially independent, such that:

$$
\chi=\Phi\left(X, u, \dot{u}, \ldots, u^{(\alpha)}\right),
$$

and:

$$
\left\{\begin{array}{l}
X=\Psi_{X}\left(\chi, \dot{\chi}, \ldots, \chi^{(\beta-1)}\right), \\
u=\Psi_{u}\left(\chi, \dot{\chi}, \ldots, \chi^{(\beta)}\right)
\end{array}\right.
$$

where $\Psi_{X}$ and $\Psi_{u}$ are smooth functions, $\chi_{i}^{(k)}$ denoting the $k^{\text {th }}$ order time derivative of the $i^{\text {th }}$ component of $\chi$, and the multi-index $\beta=\left(\beta_{1}, \ldots, \beta_{m}\right)$ contains the characteristic numbers associated to the flat outputs and is defined by::

$\beta_{i}=\min \left\{k \in \mathbb{N}^{*}: \partial\left(\frac{d^{k} \chi_{i}}{d t^{k}}\right) / \partial u_{j} \neq 0, j \in\{1, \ldots, m\}\right\}$,

with $i=1, \ldots, m$. The elements of $\chi \in \mathbb{R}^{m}$ are called flat outputs.

Since we focus attention on linear systems, we recall from Fliess et al. [1999] the following result:

Proposition 2. A linear sytem is flat if and only if it is controllable

In our case, system (4) is fully controllable since the chaser is fully actuated. One can easily check that the controllability matrix of the pair $\left(\tilde{A}_{T H}, \tilde{B}_{T H}\right)$ is full-row rank for $e<1$. Consequently, system (4) is differentially flat. Moreover, an eligible flat output is the position vector $\chi=[\tilde{x} \tilde{y} \tilde{z}]^{T}$ (see [Lévine and Ngyuen 2003, footnote 2] for complementary explanations). Thus function $\Psi$ is trivial and function $\Psi_{u}$ is obtained by inversing Tshauner Hempel equations (3): 


$$
\left\{\begin{array}{l}
\tilde{u}_{R}=\chi_{1}^{\prime \prime}-\frac{3}{1+e \cos \nu} \chi_{1}-2 \chi_{2}^{\prime} \\
\tilde{u}_{S}=\chi_{2}^{\prime \prime}+2 \chi_{1}^{\prime} \\
\tilde{u}_{W}=\chi_{3}^{\prime \prime}+\chi_{3}
\end{array}\right.
$$

The real interest of flatness for optimal control problem is that it also defined a Lie-Bäcklund equivalence between a nonlinear system and a trivial system (see Fliess et al. [1999] for explanations). As $\chi$ represent the state of the trivial system, the $m$-components of $\chi$ are differentially independent. Indeed, a $\bar{\chi}$-space of dimension $n_{\chi}$ can be considered with the coordinates $\bar{\chi}=\left\{\chi, \chi^{\prime}, \chi^{\prime \prime}, \ldots, \chi^{(p)}\right\}$ with $p \in \mathbb{N}$ where any curve of this space is equivalent to the system trajectories.

As it will be described in the next paragraph, the solution to the optimal control problem can be described as a particular curve of the $\bar{\chi}$-space.

\subsection{Flat formulation of the RdV guidance problem}

The generation of constrained trajectory consists in determining a finite-time trajectory $t \mapsto(\tilde{X}(\nu), \tilde{u}(\nu))$ with $\nu \in\left[\nu_{i} ; \nu_{f}\right]$, satisfying the set of constraints related to the dynamics of the underlying system, boundary conditions, path and actuators constraints. The problem can be formulated as follows:

$$
\min _{\tilde{u}} J(\tilde{X}, \tilde{u})\left\{\begin{array} { l } 
{ \frac { d \tilde { X } ( \nu ) } { d \nu } = \tilde { A } _ { T H } \tilde { X } ( \nu ) + \tilde { B } _ { T H } \tilde { u } ( \nu ) , } \\
{ \text { subject to: } }
\end{array} \left\{\begin{array}{l}
\tilde{X}\left(\nu_{i}\right)=\tilde{X}_{0}, \quad \tilde{u}\left(\nu_{i}\right)=\tilde{u}_{0} \\
\tilde{X}\left(\nu_{f}\right)=\tilde{X}_{f}, \quad \tilde{u}\left(\nu_{f}\right)=\tilde{u}_{f} \\
\gamma(\tilde{X}(\nu), \tilde{u}(\nu)) \geq 0
\end{array}\right.\right.
$$

where $J(\tilde{X}, \tilde{u})$ represents a particular objective function and $\gamma(\tilde{X}(\nu), \tilde{u}(\nu))$ the path and actuators constraints. The path and actuators constraints are such that:

$$
\begin{array}{r}
-\tilde{U}_{\text {max }} \leq \tilde{U}_{i} \leq \tilde{u}_{\text {max }}, \quad i=\{R, S, W\} \\
\tilde{x}^{2}+\tilde{y}^{2} \geq d^{2}
\end{array}
$$

Constraint (12) comes from the saturation on the actuators. Indeed, from equation (10), the saturation constraints may be expressed in terms of $\bar{\chi}$ :

$$
\left\{\begin{array}{l}
-\tilde{U}_{\max } \leq \chi_{1}^{\prime \prime}-\frac{3}{1+e \cos \nu} \chi_{1}-2 \chi_{2}^{\prime} \leq \tilde{U}_{\max } \\
-\tilde{U}_{\max } \leq \chi_{2}^{\prime \prime}+2 \chi_{1}^{\prime} \leq \tilde{U}_{\max } \\
-\tilde{U}_{\max } \leq \chi_{3}^{\prime \prime}+\chi_{3} \leq \tilde{U}_{\max }
\end{array}\right.
$$

Alternatively, the saturation contraint can be defined as the membership of the trajectory $\bar{\chi}(t)$ to a polytope of $O_{\bar{\chi}}$ described by its cartesian coordinates:

$$
H_{\text {sat }}(\nu) \bar{\chi}(\nu) \leq \tilde{U}_{\max } .
$$

In order to have a constant matrix $H$, the variant term $\frac{3}{1+e \cos \nu}$ is replaced by its worst case approximation:upper and lower bound $\frac{3}{1+e}$ and $\frac{3}{1-e}$ such that:

$$
H_{\text {sat }}=\left[\begin{array}{ccccccccc}
\frac{3}{1-e} & 0 & -1 & 0 & 2 & 0 & 0 & 0 & 0 \\
-\frac{3}{1+e} & 0 & 1 & 0 & -2 & 0 & 0 & 0 & 0 \\
0 & -2 & 0 & 0 & 0 & -1 & 0 & 0 & 0 \\
0 & 2 & 0 & 0 & 0 & 1 & 0 & 0 & 0 \\
0 & 0 & 0 & 0 & 0 & 0 & -1 & 0 & -1 \\
0 & 0 & 0 & 0 & 0 & 0 & 1 & 0 & 1
\end{array}\right]
$$

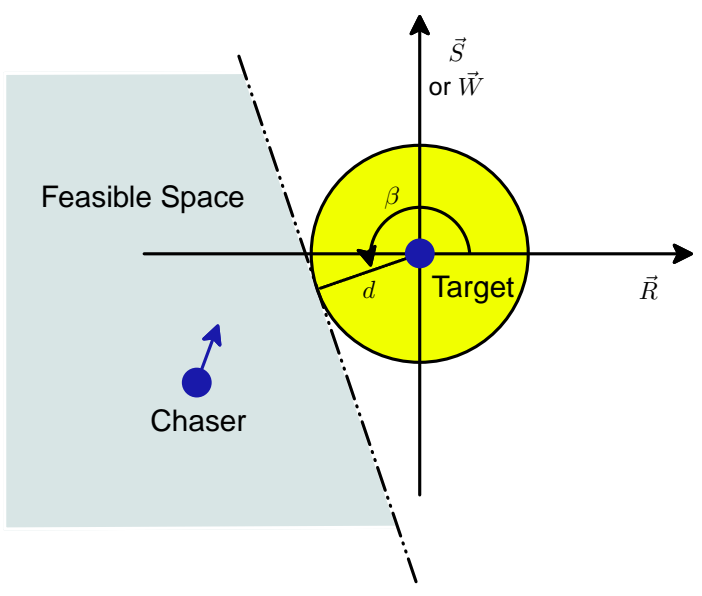

Figure 1. Convex approximation of the non convex avoidance region

Equation (13) represents the collision avoidance constraint. The definition of the collision avoidance is that a trajectory can not cross the cylindric safe zone around the target. A such constraint is given by $\tilde{x}^{2}+\tilde{y}^{2} \geq d^{2}$ that is non linear and non convex. As in Mueller and Larsson [2008], the late collision avoidance definition is replaced by the position must lie in a halfspace defined by a tangent line on the circle with the general equation $\tilde{x} \cos \beta+\tilde{y} \sin \beta=d$.

The collision avoidance constraint will then be derived as

$$
-\chi_{1} \cos \beta-\chi_{2} \sin \beta \leq-d
$$

In order to drive round the safe region, we will enforce the rotation of the half plane defining the feasible region. To achieve, the angle $\beta(\nu)$ is a piecewise constant function. The consequence is that the constraint (17) remains linear in $\chi$ and is constant piecewisely during the maneuver time.

Using the specific flatness properties, the optimal control problem (11) is transformed into the following problem:

Problem 3. Considering the flat system (6), the optimal path planning problem (11) can be formulated as the following optimization problem:

$$
\min _{\bar{\chi}} J(\bar{\chi}(\nu)) \quad\left\{\begin{array} { c } 
{ \overline { \chi } ( \nu _ { i } ) = \overline { \chi } _ { i } } \\
{ \text { subject to: } }
\end{array} \left\{\begin{array}{c}
\bar{\chi}\left(\nu_{f}\right)=\bar{\chi}_{f}, \\
\bar{\chi}(\nu) \in S_{\bar{\chi}}
\end{array}\right.\right.
$$

where $\bar{\chi}$ are flat space coordinates, $J(\bar{\chi})$ is assumed to be convex in terms of $\bar{\chi}$ and the subset $S_{\bar{\chi}}$, the so-called feasible region, is defined by equations (15) and (17). Note that, since only the second derivative of $\chi$ is involved in system (14), we will consider the $\bar{\chi}$-space $O_{\bar{\chi}}$ such that:

$$
O_{\bar{\chi}}=\left\{\chi, \chi^{\prime}, \chi^{\prime \prime}\right\}
$$

The dimension of $O_{\bar{\chi}}$ is 9 .

\subsection{B-splines Parametrization}

Then, by virtue of (18), it turns out that the optimal control problem for a flat system consists in determining a 
finite time trajectory $\nu \mapsto \chi(\nu)$ that connects two points of the $\bar{\chi}$-space and belongs to the subset $S_{\bar{\chi}}$. Since all curves of $\bar{\chi}$-space verify the nonlinear system dynamics, problem (11) is equivalent to the geometric and integration-free problem (18). One of the advantages of problem 3 is that it can be solved by all algorithms able to determine curves belonging to a well-determined subspace. The Bsplines formalism from Milam et al. [2000], Louembet et al. $[2009, ?]$ offers a convenient framework to define piecewise polynomial trajectories and constraints (particulary piecewise constant constraints) providing high flexibility with a low number of parameters. Indeed, in this paper, the trajectories of the flat output $\chi$ components and their derivatives are represented with a B-splines basis:

$$
\begin{aligned}
& \chi_{i}(\nu)=\sum_{j=1}^{n_{B}} C_{i, j} \cdot B_{j, k}(\nu), \quad i=1, \ldots, m \\
& \chi_{i}^{(r)}(\nu)=\sum_{j=1}^{n_{B}} C_{i, j} \cdot B_{j, k}^{(r)}(\nu), \quad i=1, \ldots, m .
\end{aligned}
$$

Here $\left\{B_{j, k}\right\}$ is a $k^{t h}$ order B-splines basis built on a given knot sequence $T$ (see Louembet et al. [2009] for further details on B-splines usage and [de Boor 1978, chap. VIII] for complete definitions). The control points $C_{i, j}$ are the coordinates of the piecewise polynomials $\chi_{i}(\nu)$ in the Bsplines basis.

Let $C=\left(C_{1,1}, \ldots, C_{1, n_{B}}, C_{2,1}, \ldots, C_{2, n_{B}}, C_{3,1}, \ldots, C_{3, n_{B}}\right)$ be the vector of the control points defining the trajectories $\chi(\nu)$. Using a B-splines parameterization of the flat output, the control points $C$ become the decision variables of the flat optimal control problem (18).

Problem 4. Consider flat system (6), the optimal path planning problem using B-splines parametrization can be formulated as follows:

$$
\min _{C} J(\bar{\chi}(C)) \quad\left\{\begin{array}{c}
\bar{\chi}\left(t_{i}, C\right)=\bar{\chi}_{i}, \\
\bar{\chi}\left(t_{f}, C\right)=\bar{\chi}_{f}, . \\
\bar{\chi}(C) \in S_{\bar{\chi}} .
\end{array}\right.
$$

The constraint $\bar{\chi}(C) \in S_{\bar{\chi}}$ can be seen as an inclusion of $\bar{\chi}(\nu)$ trajectories within the intersection of several halfspaces. In fact, it will be shown that positioning the trajectory $\bar{\chi}(\nu)$ in a half-space is equivalent to evaluate the sign of the piecewise polynomial gap function, $\kappa(\nu)$, between $\bar{\chi}(\nu)$ and the hyper-plane boundary. Thus, this positioning problem is equivalent to a positivity problem of piecewise polynomials. By using the concept of positive B-splines developed through LMI approach in Louembet et al. [2010], the problem 4 will be recast as a semidefinite program providing solutions, if there exist, that verify the constraint $\bar{\chi}(C) \in S_{\bar{\chi}}$ all along the path.

\section{PATH PLANNING AS A B-SPLINES POSITIVITY PROBLEM}

\subsection{Elements of piecewise polynomial positivity}

In Louembet et al. [2010], the sums of squares representation of piecewise polynomials function has been developed. This representation is convenient since it permits to link the semidefinite positiveness of piecewise polynomial with the cone of semidefinite positive matrix. In fact, this set of positive piecewise polynomial is shown to be a linear image of the cone of the positive semidefinite matrices. Let $p(t)$ be a piecewise polynomial defined on the Bsplines basis $\left\{v_{i}(t)\right\}$ such that $P(t)=\sum_{i=1}^{n_{v}} \mu_{i} v_{i}(t)$ with $\mu$ being the coordinates of $P(t)$ on $v_{i}(t)$. Through the linear operator $\Lambda^{*}$, the set of the coefficients $\mu$ may be described on a B-spline basis $v(t)$ that define a positive piecewise polynomial function.

Theorem 5. Let $\mu$ be an element of the closed, pointed and convex cone $\mathrm{K}$ defined by:

$$
\mathrm{K}=\left\{\mu \in \mathbb{R}^{n_{v}}: \mu=\Lambda^{*}(Y), Y \succeq 0\right\} .
$$

Each element $\mu$ of $\mathrm{K}$ describes a positive semidefinite polynomial on the basis $v(t)$ so that

$$
P(t)=\sum_{i=1}^{n_{v}} \mu_{i} v_{i}(t) \geq 0 .
$$

In the aim of not duplicate information available in open litterature, definitions of $\Lambda^{*}$ and proof of the theorem 5 are detailed in Louembet et al. [2010].

\subsection{Motion planning as an LMI problem}

This result, mainly based on theorem 5 , is the description of the piecewise polynomial trajectory inclusion into a polytope as a B-spline positivity problem and consequently as an LMI problem.

Le $O_{\bar{\chi}}$ be the finite dimensional flat output space with the following coordinates:

$$
\bar{\chi}=\left(\chi_{1}, \ldots, \chi_{m}, \dot{\chi}_{1}, \ldots, \dot{\chi}_{m}, \chi_{1}^{(r)}, \ldots, \chi_{m}^{(r)}\right)
$$

Recall that the flat trajectories $\left[\nu_{0}, \nu_{f}\right] \rightarrow \mathbb{R}^{n} \bar{\chi}, \nu \mapsto \chi(\nu)$ are parametrized on $k$-order B-splines basis $\left\{B_{k}\right\}$ (see equation (20)).

Let the feasible region $S_{\bar{\chi}}$ be an intersection of $n_{c}$ halfspaces of $O_{\bar{\chi}}$ and $H_{i}$ be the $i^{\text {th }}$ half-space described by its Cartesian coordinates:

$$
H_{i}=\left\{\bar{\chi} \in \mathbb{R}^{n} \bar{\chi} \mid a_{i}^{T} \bar{\chi} \leq b_{i}\right\}
$$

where $a_{i} \in \mathbb{R}^{n_{\bar{\chi}}}$ and $b_{i} \in \mathbb{R}$ are possibly piecewise polynomials in time with $i=1, \ldots, n_{c}$. We note that $\bar{\chi}(\nu)$ belong to the half-space $H_{i}$ if and only if:

$$
a_{i}^{T} \bar{\chi}(\nu) \leq b_{i}
$$

Theorem 6 . Solving the path planning problem defined by (21), is equivalent to solving the following SDP problem:

$$
\text { subject to: }\left\{\begin{array}{l}
\min _{C} J(\bar{\chi}(C)) \\
Y_{i} \succeq 0 \\
\Theta C=\theta
\end{array} \quad, \forall i=1, \ldots, n_{c} .\right.
$$

with the objective function assumed to be linear in $\bar{\chi}$ and in the control points

$$
C=\left(C_{1,1}, \ldots, C_{1, n}, C_{2,1}, \ldots, C_{2, n}, C_{3,1}, \ldots, C_{3, n}\right)
$$

as well.

$\Lambda^{*}$ is the dual operator defined in Louembet et al. [2010]. $\alpha_{i} \in \mathbb{R}^{n_{v} \times N_{C}}$ are linear matrix functions of $a_{i}$, with $a_{i}$ and $b_{i}$ associated to the $i^{t h}$ half-space $H_{i}$ (cf. equation (24)). The equality constraint $\Theta C=\theta$ represents the initial and final conditions.

A complete proof is detailed in Louembet et al. [2010]. Nevertheless an insight may be that each sum from (24) 
of weighted flatoutput trajectories and their derivatives is piecewise polynomial $\kappa_{i=1, \ldots, n_{c}}(\nu)=\sum_{j=1}^{n_{v}} \kappa_{i, j} v_{j}(\nu)$ defined on B-splines basis $v(\nu)$ such that $\kappa_{i}(\nu) \geq 0$. Finally the $\kappa$ coefficients are determined using theorem 5 .

Note that to match semidefinite program definition, the cost of problem (26) has to be linear in $C$.

\section{ORBITAL HOMING EXAMPLE}

In this section we detailed the rendezvous problem and propose a solution using the methodology presented in section 3 . The studied case is inspired by the ATV mission. The Keplerian parameters of the target orbit are given in table 1. Inclinaison, Perigee argument and RAAN parameters are omitted since a keplerian orbit is considered.

Table 1. Mission example

\begin{tabular}{c|c} 
Excentricity $e$ & 0.0052 \\
\hline Initial homing anomaly $\nu_{1}$ & 0 \\
\hline Final homing anomaly $\nu_{f}$ & $5 \mathrm{rad}$ \\
\hline Initial state $X_{1},[\mathrm{~m}, \mathrm{~m} / \mathrm{s}]$ & {$[0,50,0,0,0,0]$} \\
\hline Final state $X_{f},[\mathrm{~m}, \mathrm{~m} / \mathrm{s}]$ & {$[0,-30,0,0,0,0]$} \\
\hline Chaser's mass & $500 \mathrm{~kg}$ \\
\hline Actuator saturation & $1 \mathrm{~N}$
\end{tabular}

The trajectory $t \mapsto \chi(\nu)$ is a $5^{t h}$ order piecewise polynomial function defined on the sequence of equidistant knots $\xi=\left\{\xi_{1}, \ldots, \xi_{15}\right\}$. Indeed $\chi(\nu)$ admits $B(\nu)$ as B-splines basis such that the trajectory can handle non zero initial and final conditions at $\left(\xi_{1}\right.$ and $\left.\xi_{15}\right)$ and has the highest continuity degree at interior breakpoints $\left(\xi_{2}\right.$ to $\left.\xi_{14}\right)$ i.e 4 .

The number, $n_{c}$, of linear constraints in $\bar{\chi}$, is $7: 6$ for the saturation contraints (equation (15)) and 1 for the collision avoidance (equation (17)). In order to define $\Lambda^{*}$, we need to characterize the gap functions $\kappa_{i}(\nu)$. Since the higher derivation order involved in saturation constraints (14) is two, the $\kappa_{i=1 \ldots 6}(\nu)$ are fifth order piecewise polynomials of continuity class $C^{0}$ at initial and final knots $\left\{\xi_{1}, \xi_{f}\right\}$ and $C^{2}$ at the interior knots $\left\{\xi_{2}, \ldots, \xi_{14}\right\}$.

The constraint (17) involves linearly only the flat outputs but has piecewise constant coefficient. In fact, in order to enforce the bypass of the safe region, the angle $\beta$ in (17) is piecewise constant fonction of time such that:

$$
\beta=\beta_{1}+i\left(\frac{\beta_{f}-\beta_{1}}{7}\right) \text {, if } \nu \in\left[\xi_{2 i+1}, \xi_{2 i+3}\right], i=0 \ldots 6
$$

$\beta_{1}$ and $\beta_{f}$ are the direction angle to initial and final position in the orbit plane:

$$
\beta_{1}=\arctan \left(\frac{\tilde{X}_{1, S}}{\tilde{X}_{1, R}}\right), \beta_{f}=\arctan \left(\frac{\tilde{X}_{f, S}}{\tilde{X}_{f, R}}\right)
$$

Thus, the gap function $\kappa_{7}(\nu)$ is $C^{0}$ at the odd knots $\xi_{1}, \xi_{3}, \ldots, \xi_{15}$ and $C^{4}$ at even knots $\xi_{2}, \xi_{4}, \ldots, \xi_{14}$. The operators $\Lambda_{i}^{*}$ and coefficient matrices $\alpha_{i}$ of problem (26) are then calculated using methodology developped in Louembet et al. [2010].

Problem (26) is solved using Yalmip from Löfberg [2004] and Sedumi 1.02 from Sturm [1999].

The obtained trajectory is given in figure 1 . Figure 2 shows that the in-plane trajectory respects clearly the avoidance constraints.

For sake of comparison, we solve problem (21) by means of flatness and collocation methods described in Louembet et al. [2009], Milam et al. [2000], Faiz et al. [2001]. Recall that the constraints are checked in a finite number of time called collocation points. The problem is solved with the quadratic solver MATLAB quadprog considering 25 collocation points that are equidistant in anomaly. Although an admissible solution for the collocation problem is quickly obtained, the trajectory violates the constraints between the collocation points: the avoidance contraints for $\beta=$ $\frac{5 \pi}{6}, \pi, \frac{7 \pi}{6}$ are obviously transgressed and constraints for $\beta=\frac{8 \pi}{6}, \pi, \frac{3 \pi}{2}$ are also violated see figure 3 . Moreover, we can observe on figure 3 that our methodology can produce trajectories very close to the bounds without violating them. On the contrary, when collocation points get closed to the boundaries, the situation could lead to constraints violation. Thrust profiles along $R$ and $S$ direction are presented on figure 4 . Both thrust profiles are equivalent and not saturated and the cost objectives are less than $3 \%$ different. With the above collocation methods, an iterative process is needed to re-distribute the sequence or increase the number the collocation points. This is to be compared to the one-shot method exposed in this paper.

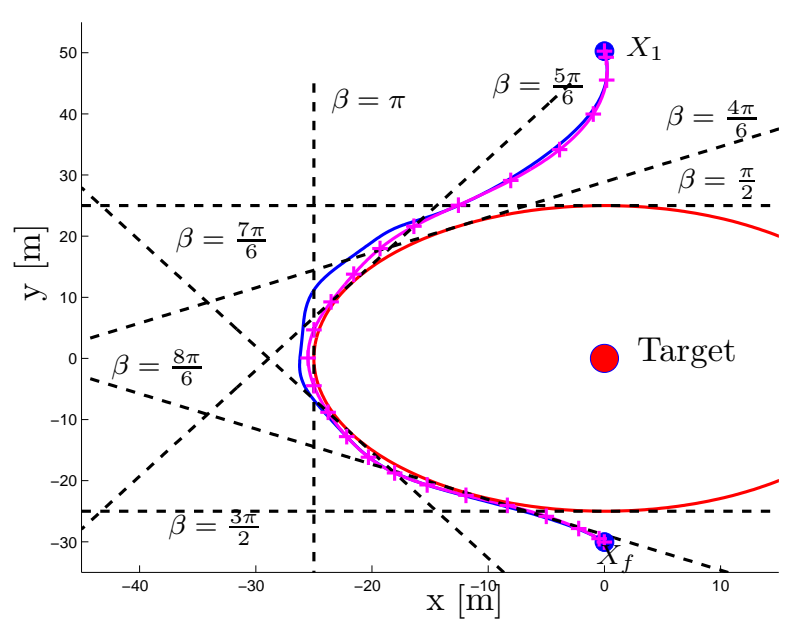

Figure 2. Trajectories $\bar{\chi}(t)$ obtained by SDP (blue) and by collocation (magenta), the collocation points are the crosses point, red circle is the safe region, dash line are the sequential approximation of the safe zone

\section{CONCLUDING REMARKS}

In this paper, the orbital rendezvous planning problem using continuous thrust is solved by means of a new approach based on the differential flatness and positive piecewise polynomials results. As opposed to most works on direct methods for optimal control problem reported in the literature, the developed methodology provides a new framework for satisfying constraints all along the path.

\section{REFERENCES}

Anzel, B.M. (1998). Stationkeeping the hughes hs 702 satellite with a xenon ion propulsion system. In 49th International Astronautical Congress. Melbourne, Australia. 


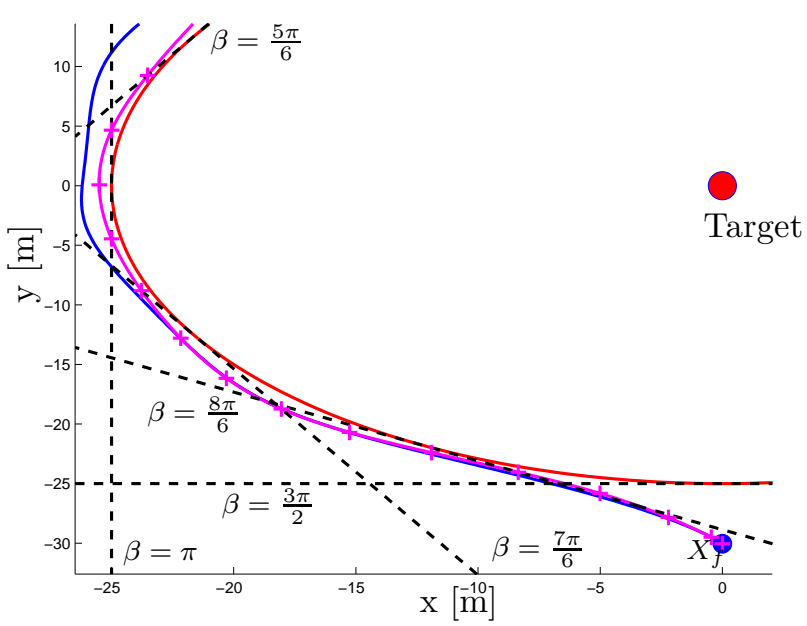

Figure 3. Focus
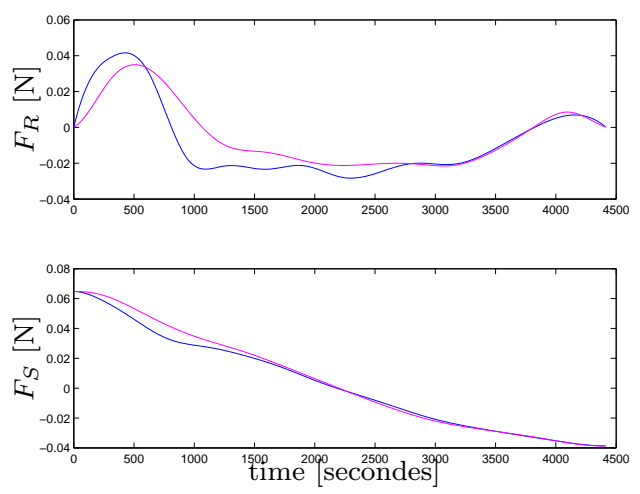

Figure 4. Thrust profiles

Anzel, B. (1988). Controlling a stationary orbit using electric propulsion. In DGLR/AIAA/JSASS 20th International Electric propulsion Conference. GarmischPartenkirchen, Germany.

Armellin, R., Massari, M., and Finzi, A. (2004). Optimal Formation Flying Reconfiguration and Station Keeping Maneuvers Using Low Thrust Propulsion. In 18th International Symposium on Space Flight Dynamics, volume 548, 429. Munich, Germany: Oct.

Breger, L. and How, J. (2008). Safe trajectories for autonomous rendezvous of spacecraft. Journal of Guidance, Control and Dynamics, 31(5), 1478-1489.

de Boor, C. (1978). A pratical guide to spline. In Applied Mathematical Sciences, volume 27. Springer.

Eckstein, M., Rajasingh, C., and Blumer, P. (1989). Colocation strategy and collision avoidance for the geostationary satellites at 19 degrees west. In International Symposium on Space Flight Dynamics, volume 6.

Faiz, N., Agrawal, S., and Murray, R. (2001). Differentially flat systems with inequality constraints: An approach to real-time feasible trajectory generation. J. Guidance, Control and Dynamics, 24.

Fliess, M., Lévine, J., Martin, P., and Rouchon, P. (1992). On differentially flat nonliear systems. Proc. 2nd. IFAC NOLCOS Symposium Bordeaux.

Fliess, M., Lévine, J., Martin, P., and Rouchon, P. (1999). a lie-bäcklund approach to equivalence and flatness of nonlinear systems. IEEE Trans. Automat. Contr., 44(5),
922-937.

Gil-Fernndez, J., Milic, E., Graziano, M., Polle, B., Mancuso, S., and Fertig, J. (2006). Autonomous low-thrust guidance scheme for interplanetary trajectories: Application to smart-1. In E.S. Agency (ed.), 6th International ESA Conference on Guidance, Navigation and Control Systems. Loutraki, Greece.

Gopinath, N.S. and Srinivasamuthy, K.N. (2003). Optimal low thrust orbit transfer from gto to geosynchronous orbit and stationkeeping using electric propulsion system. In 54th International Astronautical Congress of the International Astronautical Federation, the International Academy of Astronautics, and the International Institute of Space Law. Bremen, Germany.

Hull, D.G. (1997). Conversion of optimal control problems into parameter optimization problems. J. Guidance, Control and Dynamics, 20(1).

Junge, O. and Ober-Blobaum, S. (2005). Optimal reconfiguration of formation flying satellites. In 44th IEEE Conference on Decision and Control, 2005 and 2005 European Control Conference. CDC-ECC'05, 66-71.

Lévine, J. and Ngyuen, D. (2003). Flat output characterisation for linear systems using polynomial matrices. Systems and Control Letters, 48, 69-75.

Löfberg, J. (2004). Yalmip : A toolbox for modeling and optimization in MATLAB. In Proceedings of the CACSD Conference. Taipei, Taiwan. URL http://control.ee.ethz.ch/ joloef/yalmip.php.

Louembet, C., Cazaurang, F., and Zolghadri, A. (2010). Motion planning for flat systems using B-splines parametrization:an LMI approach. Automatica, 46, 1305-1309.

Louembet, C., Cazaurang, F., Zolghadri, A., Pittet, C., and Charbonnel, C. (2009). Path planning for satellite slew maneuvers: A combined flatness and collocation based approach. IET Control Theory \& Applications.

Milam, M., Mushambi, K., and Murray, R. (2000). A new computational approach to real-time trajectory generation for constrained mechanical systems. In IEEE Conference on Decision and Control.

Mueller, J. and Larsson, R. (2008). Collision avoidance maneuver planning with robust optimization. In $7^{t} h$ International ESA Conference on Guidance, Navigation $\&$ Control Systems. Tralee, County Kerry, Ireland.

Rayman, M., Chadbourne, P., Culwell, J., and William, S. (1999). Mission design for deep space 1: A low-thrust technology validation mission. Acta Astronautica, 45(49), 381-388.

Richards, A., Schouwenaars, T., How, J., and Feron, E. (2002). Spacecraft trajectory planning with avoidance constraints using mixed-integer linear programming. Journal of Guidance, Control, and Dynamics, 25(4), $755-764$.

Sturm, J. (1999). Using SeDuMi 1.02, a Matlab toolbox for optimization over symmetric cones. Optimization methods and software, 11-12, 625-653.

Tschauner, J. (1966). The elliptic orbit rendezvous. In AIAA 4th Aerospace Sciences Meeting. Los Angeles, Californie, USA.

Wang, S. and Schaub, H. (2010). Electrostatic Spacecraft Collision Avoidance Using Piecewise-Constant Charges. Journal of guidance, control, and dynamics, 33(2). 